\title{
Structural valve deterioration does not alter tissue valves' radiopaque landmarks: implications for valve-in-valve therapy
}

\author{
Réda BELHAJ SOULAMI ${ }^{1,2,3}$, Miguel CASTRO ${ }^{1,2}$, Pascal HAIGRON ${ }^{1,2}$, Jean-Philippe VERHOYE ${ }^{1,2,3}$ \\ ${ }^{1}$ INSERM, UMR 1099, Rennes F-35000, France \\ ${ }^{2}$ Université de Rennes 1, LTSI, Rennes, F-35000, France \\ ${ }^{3}$ Department of thoracic and cardiovascular Surgery, Pontchaillou University Hospital - Rennes, France
}

Corresponding author: Réda BELHAJ SOULAMI, Service de chirurgie thoracique et cardiovasculaire, CHU de Rennes, 2 Rue Henri Le Guilloux, 35000 Rennes. Mail: belhajreda@hotmail.com. Phone: 0033299282595

Acknowledgments: This work has been supported by the ADETEC association. 


\section{SUMMARY}

Background Valve-in-valve is established as a safe and efficient alternative to redo surgery in the treatment of structural valve deterioration (SVD). In vitro models rely on the radiopaque landmarks of undeteriorated tissue valves to establish the optimal implantation level of the transcatheter heart valves inside the deteriorated valves. In computed assisted procedures, the radiopaque landmarks of the deteriorated valves may be used to guide valve implantation through image fusion. The purpose of this study is to determine whether SVD alters the radiopaque landmarks of stented tissue valves.

Methods Our approach was based on the computation of relevant anatomical measurements from CT images. Radiopaque landmarks of degenerated bioprostheses and the corresponding undeteriorated valves were extracted to create surface meshes and cloud points using grey-level thresholding. 3D registration using an iterative closest point algorithm was used to align the corresponding cloud points, while the modified Hausdorff Distance was applied to determine the differences between them.

Results The proposed evaluation was performed on 19 degenerated tissue valves. 15 valves were scanned from patients evaluated for valve-in-valve procedures, and 4 bioprostheses were scanned after surgical extraction during redo aortic valve replacement. All the degenerated valves were compared to the corresponding undeteriorated models. Overall, the mean difference between degenerated and undeteriorated valves was $0.33+/-0.12 \mathrm{~mm}$. The maximum observed registration error was $0.66 \mathrm{~mm}$.

Conclusions Our study demonstrates no significant difference between the radiopaque landmarks of deteriorated and undeteriorated bioprostheses after the occurrence of SVD. Our findings suggest therefore that SVD does not alter radiopaque landmarks of stented tissue valves. These results validate in-vitro studies of optimal transcatheter heart valves implantation inside deteriorated tissue valves based on their radiopaque landmarks, and allow the use of non-deteriorated valves' imaging features in computer assisted valve-in-valve procedures. 


\section{INTRODUCTION}

The vast majority of heart valve replacements are currently performed using stented bioprostheses [1]. Compared to mechanical valves, tissue valves offer lower thrombogenicity and avoid long-term anticoagulation. However, tissue valves are prone to structural valve deterioration (SVD), resulting in limited long-term durability [1,2]. SVD manifests as the alteration of the tissue valve's leaflets, either involving calcification and stenosis or tear and regurgitation, or both [1]. In this context, the Valve-in-valve (ViV) technique is now established as a safe and efficient alternative to redo surgical valve replacement in the management of failing tissue valves [3]. ViV consists in the implantation of a transcatheter heart valve (THV) inside a degenerated tissue valve, under fluoroscopic guidance (Figure 1). The THV's level (or depth) of implantation inside the deteriorated tissue valve is of paramount importance for the attainment of optimal hemodynamics [4]. Such level is determined based on the deteriorated valve's radiopaque landmarks, corresponding to the valve's stent. Suboptimal implantation may also lead to complications such as device embolization, coronary obstruction, periprosthetic regurgitation or mitral valve injury [5].

Several in-vitro studies have in fact demonstrated, for various combinations of THVs and bioprostheses, the optimal level of implantation of the THV inside the tissue valve [4, 6, 7]. Fluoroscopic guidelines [8] for an ideal implantation have also been suggested. However, these benchmarks rely on new, undeteriorated tissue valves, and therefore infer that SVD does not alter the tissue valve's stent, and therefore its radiopaque landmarks. Nevertheless, SVD occurs typically between 10 and 20 years after implantation of the tissue valve (473.040.000 heartbeats in 15 years at an average 60 beats per minute). Given the dynamic architecture of the aortic root [9], and the postsurgical changes in aortic wall shear stress after aortic valve replacement (AVR) [10], the statement that valve's stent remains undistorted throughout time has to be challenged.

Moreover, we have previously demonstrated the feasibility of computer assisted aortic ViV procedures [11]. Our approach was based on the segmentation of the patients' deteriorated tissue valve from the preoperative CT-scan, creating a surface mesh that was registered on the fluoroscopic image. However, image segmentation from patients' CT-scan can be challenging in the case of imaging artifacts, poor-quality or non ECG-gated CT-scans, and in the case of tissue valves with limited radiopaque landmarks. In the case of computer assisted ViV procedures, the use of surface meshes extracted from undeteriorated bioprostheses would simplify the image-based assistance, allowing a direct registration of the corresponding undeteriorated tissue valve with the pre-determined optimal implantation level. 


\section{THE HYPOTHESIS}

The purpose of this study is to evaluate whether SVD alters the radiopaque landmarks of stented tissue valves. While it has been demonstrated that SVD alters the valve's leaflets, little is known of the valve's structure, including its radiopaque landmarks, often after more than a decade of heartbeats.

\section{METHODS}

\section{Overall Workflow}

This was a retrospective observational study, based on the comparison of the radio-opaque landmarks of deteriorated tissue valves and the corresponding undeteriorated models. The evaluation was based on the computational analysis of CT-scan images. The information of the degenerated biological valves stent (BVS) was extracted either from pre-operative CT-scans of patients undergoing a ViV procedure, or from CT-scans of surgically extracted degenerated tissue valves after redo AVR. The undeteriorated corresponding bioprostheses were scanned while sealed in their original packaging at room temperature. Each BVS was segmented and represented as a surface mesh.

\section{Stented tissue valves description}

The design of tissue valves aims towards mimicking the anatomy of the native aortic valve [12]. Porcine valves consist of 3 porcine aortic valve leaflets, while pericardial valves are made from sheets of bovine pericardium, mounted inside or outside a supporting stent. Valve stents are usually metallic or fabricated from a variety of flexible polymers. The ViV procedure involves the implantation of a THV inside the degenerated tissue valve, based on the tissue valve's radiopaque features (Figure 1). Such landmarks correspond to the valve's stent, additionally to the sewing cuff or stent posts in some models.

\section{BVS Segmentation and Creation of Cloud Points and Surface Meshes}

A binary image was created from the original Region of interest (ROI) from CT images by applying a gray-level thresholding method using ITK-Snap software [13]. In the case of pre-ViV scans, calcifications and artifacts were removed manually. A surface mesh was then created based on the edge points of the binary image. The surface mesh was created for visualization purposes. Its vertices were considered as cloud points, used in the registration method and in the comparison metric. 


\section{D Rigid registration algorithms}

Image registration refers to the determination of one to one correspondence between the coordinates of two or more images such as each corresponding point to refer to the same anatomical point. When the images and the objects that they represent have different scaling, then the images are co-registered, aligned, and geometrically transformed. One of the images is used as reference to the geometric transformation that is applied in the other images (source images) so that they finally align with the reference. In this study, iterative closest point (ICP) algorithm was used in all the valves except the Medtronic Mosaic (Medtronic, Minneapolis, US) heart valve. In this particular case, point-based registration with known correspondence was used given the radiopaque features of this valve (three radiopaque markers at the top of each stent post) (figure 2).

ICP algorithm: The ICP algorithm is an efficient algorithm for robust rigid registration of 3D data. It is commonly used in medical image registration $[14,15]$. The main idea of the ICP algorithm is to search the closest points in the fixed cloud points for all moving cloud points and transform the moving cloud points iteratively. The ICP algorithm iteratively computes the new corresponding relationship and new transformation until the distance between corresponding points is not decreasing anymore or the iteration reaches the maximum iteration number $[14,15]$.

Point-based registration with known correspondence: The goal of this type of registration is to align two sets of points with the same number of corresponding points using a selected transformation type [16], in our case a rigid transformation. A known correspondence means that the $i$-th point from the first set of points corresponds to the $i$-th point from the second set of points. This approach attempts to find the correspondence using distinct landmarks that are extracted from images.

\section{Comparison metrics}

The Modified Hausdorff Distance (MHD) is used for matching two objects based on their edge points. Its value increases monotonically as the amount of difference between the two sets of edge points increases, and it is robust to outlier points that might result from segmentation errors [17]. 
Modified Hausdorff Distance: Given two finite point sets $A=\left\{\begin{array}{ll}a_{1}, a_{2}, \ldots, a_{m}\end{array}\right\}$ representing the model (unimplated BVS), and $B=\left\{b_{1}, b_{2}, \ldots, b_{k}\right\}$ representing the edge points of the degenerated BVS. The MHD is defined as:

$$
d_{M H D}(A, B)=\frac{1}{n} \sum_{a_{i} \in A} \min _{b_{j} \in B}\left\|a_{i}-b_{j}\right\|
$$

where $n$ is the number of point in $A$. This measure indicates the degree of similarity between two point sets. The MHD is more appropriate than other distance measurements for object matching purposes based on their edge points $[18,19]$.

\section{RESULTS}

The proposed evaluation was performed on two datasets (Table 1). The first group involved data of 15 degenerated tissue valves scanned from patients undergoing a ViV procedure. The second involved 4 deteriorated tissue valves extracted surgically during redo surgical aortic valve replacement. The dataset of all analyzed valves is summarized in table 1 .

Three measures have been used to evaluate and visualize the result of the alignment of the meshes: local surface differences, volume-to-volume overlap measure and point-to-surface distance.

\section{Comparison Outcomes}

\section{Medtronic Mosaic valve}

The only radio-opaque parts of the Medtronic Mosaic valve are the eyelets at the superior aspect of the stent posts (Figure 2). In this kind of bioprostheses, the three eyelets were manually marked on each CT-scan, and the alignment of the bioprostheses was based on these landmarks using a point-based registration method. Table 2 shows the root-mean-square (RMS) of the pairwise differences of the two landmarks sets. The gap between the landmarks shows the degree of deformation for this type of bioprostheses. Overall, the main RMS error for the Medtronic Mosaic Valve was $0.495 \mathrm{~mm}$, while the maximal error reached $0.663 \mathrm{~mm}$ (Table 2). 


\section{All other prostheses}

The remaining evaluated valves are characterized by a clearly visible radiopaque structure (Figures 3-6). The alignment of the bioprostheses is based on the ICP algorithm. The parameters of the registration method have been adjusted as follows: maximum number of iteration was fixed at 50 and the RMS differences less than 1.0e5. The degenerated BVS mesh is defined as the fixed cloud points and the new bioprosthesis mesh model is defined as the moving cloud. In this way, the problems due to artifacts, calcifications, motion and/or scatter (coming from the CT-scan of the degenerated valves), have less influence on the registration result. The comparison metric MHD is defined from points coming from the undeteriorated BVS to the surfacic cloud points coming from the degenerated BVS, for the same reasons that we explained above. If the measurement is made the other way (degenerated to undeteriorated), all points belonging to the artifacts would increase the error measure.

In this group, evaluated with the MHD method, the mean difference between degenerated and undeteriorated valves was $0.35+/-0.1 \mathrm{~mm}$, and the maximal difference was $0.369 \mathrm{~mm}$. The results are summarized in table 3 .

Specifically, the mean MHD was $0.289+/-0.022 \mathrm{~mm}$ was the Carpentier Edwards Magna Ease valve (Edwards, Irvine, CA) (Figure 3), $0.326+/-0.00025 \mathrm{~mm}$ for the Saint Jude Medical Trifecta (Saint Jude Medical, Saint Paul, MIN) (Figure 4), $0.264+/-0.021 \mathrm{~mm}$ for the Saint Jude Medical Epic valve (Figure 5), and $0.364+/$ $0.005 \mathrm{~mm}$ for the Carpentier Edwards Perimount valve (Figure 6).

Finally, regarding the surgically extracted degenerated tissue valves (table 4), the mean MHD was $0.139+/-$ $0.021 \mathrm{~mm}$, and the maximal MHD was $0.164 \mathrm{~mm}$.

\section{DISCUSSION}

During valve-in-valve procedures, the precision of THV implantation inside the deteriorated tissue valve impacts hemodynamic outcomes, determines potential procedural complications, and may even impact the THV's durability [5]. The implantation is guided using the radio-opaque landmarks of both the degenerated surgical valve, as well as the THV. Using a variety of THVs and tissue valves, several studies sought to determine, the ideal level of implantation of the THV inside each type of tissue valve, based on their radiopaque landmarks $[4,6,7]$. 
Therefore, it is important to assess whether SVD may alter the bioprostheses' stent, and therefore, radiopaque landmarks. Given that the placement of a THV inside a degenerated tissue valve ideally requires millimeter accuracy, we considered a registration error over 1 millimeter to be significant enough to potentially infer deformation of the radiopaque landmarks.

Among 19 degenerated tissue valves compared to undeteriorated matching valves, we observed a mean difference of $0.35+/-0.1 \mathrm{~mm}$ using an ICP $3 \mathrm{D}$ registration algorithm, point based registration with known correspondence and the MHD to determine the potential difference. The maximum observed registration error was $0.66 \mathrm{~mm}$. Such findings may even be over-estimated by registration errors subsequent to suboptimal CTscan quality and imaging artefacts.

Regarding image comparison, Dice score and Jaccard index are widely used in medical image registration, but they are not a proper distance metrics (they do not obey the triangular inequality). These metrics can be sensitive to outliers (additional small segmented objects outside the main object), class imbalance (size of the segmented object relative to the background), and number of segmented objects[20,21]. Hence, the modified Haussdorff distance, was used, described for matching two objects based on their edge points.

Finally, computer assistance in $\mathrm{ViV}$ procedures may rely on the 3D/2D registration of surface meshes extracted from the segmentation of pre-operative CT-scans onto the fluoroscopic image [11]. This semi-automated step can however be challenging in the case of imaging artifacts, poor-quality or non ECG-gated CT-scans, and in the case of tissue valves with limited radiopaque landmarks, such as the Medtronic Mosaic or the Saint Jude Trifecta valves. Our study suggests therefore that this initial step can be avoided by using surface meshes extracted from undeteriorated corresponding valves. Such valves can in fact easily be scanned, allowing the creation of a database of surface meshes for computer-assisted ViV procedures.

In the setting of computer-assisted ViV procedures, the proposed approach relies on the radiopaque landmarks of the tissue valves for image registration and dynamic tracking [11]. Stentless valves represent therefore a further complex issue due to the lack of radiopaque landmarks. Equally, evaluation of structural alterations of such valves may therefore be more challenging in order to assess the impact of structural valve deterioration.

\section{CONSEQUENCES OF THE HYPOTHESIS}

Our study suggests that SVD does not alter the stent of the bioprosthetic heart valves, and therefore does not modify their radiopaque landmarks. Such findings validate the in-vitro models studying THV implantation 
inside deteriorated tissue valves using new undeteriorated ones. Moreover, in computer-assisted procedures, it allows the use of undeteriorated valves to create image-based softwares to facilitate ViV procedures.

Acknowledgments This work has been supported by the ADETEC association. The ADETEC association had no part in manuscript writing, data collection or analysis.

\section{Compliance with ethical standards}

Conflict of interest statement: The authors declare that they have no competing interests.

Ethical standards: Anonymous retrospective procedure data were used. All patients gave consent for acceptance of both the procedure and the management of data with research purposes.

\section{REFERENCES}

1. Dvir D, Bourguignon T, Otto CM, Hahn RT, Rosenhek R, Webb JG, Treede H, Sarano ME, Feldman T, Wijeysundera HC, Topilsky Y, Aupart M, Reardon MJ, Mackensen GB, Szeto WY, Kornowski R, Gammie JS, Yoganathan AP, Arbel Y, Borger MA, Simonato M, Reisman M, Makkar RR, Abizaid A, McCabe JM, Dahle G, Aldea GS, Leipsic J, Pibarot P, Moat NE, Mack MJ, Kappetein AP, Leon MB; VIVID (Valve in Valve International Data) Investigators. Standardized Definition of Structural Valve Degeneration for Surgical and Transcatheter Bioprosthetic Aortic Valves. Circulation. 2018 Jan 23;137(4):388-99.

2. Capodanno D, Petronio AS, Prendergast B, Eltchaninoff H, Vahanian A, Modine T, Lancellotti P, Sondergaard L, Ludman PF, Tamburino C, Piazza N, Hancock J, Mehilli J, Byrne RA, Baumbach A, Kappetein AP, Windecker S, Bax J, Haude M. Standardized definitions of structural deterioration and valve failure in assessing long-term durability of transcatheter and surgical aortic bioprosthetic valves: a consensus statement from the European Association of Percutaneous Cardiovascular Interventions (EAPCI) endorsed by the European Society of Cardiology (ESC) and the European Association for Cardio-Thoracic Surgery (EACTS). Eur Heart J. 2017 Dec 1;38(45):3382-90.

3. Tam DY, Vo TX, Wijeysundera HC, Dvir D, Friedrich JO, Fremes SE. Transcatheter valve-in-valve versus redo surgical aortic valve replacement for the treatment of degenerated bioprosthetic aortic valve: A systematic review and meta-analysis. Catheter Cardiovasc Interv [Internet]. 2018 Jul 19 [cited 2018 Aug 24]; Available from: http://doi.wiley.com/10.1002/ccd.27686

4. Azadani AN, Reardon M, Simonato M, Aldea G, Nickenig G, Kornowski R, Dvir D. Effect of transcatheter aortic valve size and position on valve-in-valve hemodynamics: An in vitro study. J Thorac Cardiovasc Surg. 2017 Jun;153(6):1303-1315.e1.

5. Bapat V. Technical pitfalls and tips for the valve-in-valve procedure. Ann Cardiothorac Surg. 2017 Sep;6(5):541-52.

6. Simonato M, Azadani AN, Webb J, Leipsic J, Kornowski R, Vahanian A, Wood D, Piazza N, Kodali S, Ye J, Whisenant B, Gaia D, Aziz M, Pasala T, Mehilli J, Wijeysundera HC, Tchetche D, Moat N, Teles R, Petronio AS, Hildick-Smith D, Landes U, Windecker S, Arbel Y, Mendiz O, Makkar R, Tseng E, Dvir D. In vitro evaluation of implantation depth in valve-in-valve using different transcatheter heart valves. EuroIntervention. 2016 Sep;12(7):909_ 17. 
7. Midha PA, Raghav V, Condado JF, Okafor IU, Lerakis S, Thourani VH, Babaliaros V, Yoganathan AP. Valve Type, Size, and Deployment Location Affect Hemodynamics in an In Vitro Valve-in-Valve Model. JACC Cardiovasc Interv. 2016 Aug;9(15):1618-28.

8. Bapat VN, Attia RQ, Condemi F, Visagan R, Guthrie M, Sunni S, Thomas M.. Fluoroscopic Guide to an Ideal Implant Position for Sapien XT and CoreValve During a Valve-in-Valve Procedure. JACC Cardiovasc Interv. 2013 Nov;6(11):1186-94.

9. Lansac E. A four-dimensional study of the aortic root dynamics. Eur J Cardiothorac Surg. 2002 Oct;22(4):497-503.

10. Bollache E, Fedak PWM, van Ooij P, Rahman O, Malaisrie SC, McCarthy PM, Carr JC, Powell A, Collins JD, Markl M, Barker AJ. Perioperative evaluation of regional aortic wall shear stress patterns in patients undergoing aortic valve and/or proximal thoracic aortic replacement. J Thorac Cardiovasc Surg. 2018 Jun;155(6):2277-2286.e2.

11. Belhaj Soulami R, Verhoye JP, Nguyen Duc H, Castro M, Auffret V, Anselmi A, Haigron P, Ruggieri VG. ComputerAssisted Transcatheter Heart Valve Implantation in Valve-in-Valve Procedures. Innov Technol Tech Cardiothorac Vasc Surg. 2016;11(3):193-200.

12. Pibarot P, Dumesnil JG. Prosthetic Heart Valves: Selection of the Optimal Prosthesis and Long-Term Management. Circulation. 2009 Feb 9;119(7):1034-48.

13. Yushkevich PA, Piven J, Hazlett HC, Smith RG, Ho S, Gee JC, Gerig G. User-guided 3D active contour segmentation of anatomical structures: Significantly improved efficiency and reliability. Neuroimage 2006 Jul 1;31(3):1116-28.

14. PJ Besl, ND McKay. A method for registration of 3-D shapes. IEEE Transactions on pattern analysis and machine intelligence, 14(2):239-256, 1992

15. M Pan, J Tang, Q Rong, F Zhang. Medical image registration using modified iterative closest points. Int. J. Numer. Meth. Biomed. Engng. 27: 1150-1166, 2011

16. Kim HS, Park SB, Lo SS, Monroe JI, Sohn JW. Bidirectional local distance measure for comparing segmentations. Med Phys. 2012 Nov;39(11):6779-90

17. MP Dubuisson, AK. Jain. A modified Hausdorff distance for object matching. ICPR, 1994.

18. Fitzgibbon, A. Robust registration of $2 \mathrm{D}$ and 3D point sets. Computer Vision and Image Understanding 2003. (2): $1145-1153$.

19. Aziz Taha A, Hanbury A. Metrics for evaluating 3D medical image segmentation: analysis, selection, and tool. BMC Med Imaging. 2015; 15: 29.

20. R. C. Veltkamp. "Shape Matching: Similarity Measures and Algorithms”. In: Shape Modeling International. 2001, pp. 188-197.

21. Škrinjar O. Point-Based Registration with Known Correspondence: Closed Form Optimal Solutions and Properties. Biomedical Image Registration. WBIR 2006. LNCS, vol 4057. Springer, Berlin, Heidelberg 2006. 


\section{TABLES}

\begin{tabular}{|c|c|c|c|c|}
\hline \multicolumn{3}{|r|}{ Valve Model } & Diameter $(\mathrm{mm})$ & Number of valves \\
\hline \multirow{7}{*}{ 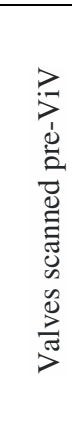 } & \multirow{7}{*}{ 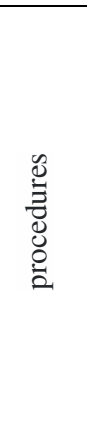 } & Medtronic Mosaic & 23 & 3 \\
\hline & & Medtronic Mosaic & 27 & 2 \\
\hline & & Carpentier Edwards Perimount Magna Ease & 21 & 3 \\
\hline & & Carpentier Edwards Perimount Magna Ease & 23 & 1 \\
\hline & & Saint Jude Trifecta & 23 & 2 \\
\hline & & Saint Jude Epic & 21 & 2 \\
\hline & & Carpentier Edwards Perimount & 25 & 2 \\
\hline \multirow{4}{*}{ 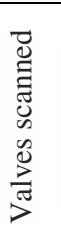 } & \multirow{4}{*}{ 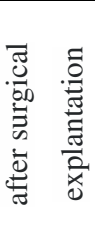 } & Medtronic Mosaic & 27 & 1 \\
\hline & & Carpentier Edwards Perimount & 23 & 1 \\
\hline & & Carpentier Edwards Supra annular & 21 & 1 \\
\hline & & Carpentier Edwards Supra annular & 23 & 1 \\
\hline
\end{tabular}

Table 1. Description of the evaluated degenerated tissue valves (ViV = Valve-in-valve).

\begin{tabular}{|l|c|}
\hline \multicolumn{1}{|c|}{ Model } & $\begin{array}{c}\text { Registration error } \\
\text { RMS (mm) }\end{array}$ \\
\hline Mosaic 23 (A) & 0.3906 \\
\hline Mosaic 23 (B) & 0.4857 \\
\hline Mosaic 23 (C) & 0.4019 \\
\hline Mosaic 27 (A) & 0.6632 \\
\hline Mosaic 27 (B) & 0.5341 \\
\hline
\end{tabular}


Table 2. Registration errors using the point-based registration with known correspondence of the Medtronic Mosaic valve.

\begin{tabular}{|l|c|c|}
\hline \multicolumn{1}{|c|}{ Model } & $\begin{array}{c}\text { Registration error } \\
\text { RMS (mm) }\end{array}$ & $\begin{array}{c}\text { MHD distance } \\
\text { unsigned mean +std (mm) }\end{array}$ \\
\hline Magna Ease 21mm (A) & 0.3202 & $0.2614 \pm 0.1867$ \\
\hline Magna Ease 21mm (B) & 0.3579 & $0.2999 \pm 0.2001$ \\
\hline Magna Ease 21mm (C) & 0.4009 & $0.3212 \pm 0.2480$ \\
\hline Magna Ease 23mm (D) & 0.3397 & $0.2752 \pm 0.2188$ \\
\hline Trifecta 23mm (A) & 0.4440 & $0.3269 \pm 0.2899$ \\
\hline Trifecta 23mm (B) & 0.4860 & $0.3264 \pm 0.2904$ \\
\hline Epic 21mm (A) & 0.28857 & $0.2425 \pm 0.1597$ \\
\hline Epic 21mm (B) & 0.34517 & $0.2856 \pm 0.2304$ \\
\hline Perimount 25 (A) & 0.4424 & $0.3595 \pm 0.2610$ \\
\hline Perimount 25 (B) & 0.4479 & $0.3696 \pm 0.2697$ \\
\hline
\end{tabular}

Table 3. Registration error using ICP (RMS) and MHD methods in degenerated versus undeteriorated tissue valves.

\begin{tabular}{|l|c|c|}
\hline \multicolumn{1}{|c|}{ Model } & $\begin{array}{c}\text { Registration error } \\
\text { RMS (mm) }\end{array}$ & \multicolumn{1}{c|}{$\begin{array}{c}\text { MHD distance } \\
\text { unsigned mean +std (mm) }\end{array}$} \\
\hline Mosaic & 0.3633 & $\begin{array}{l}\text { Registration based on } \\
\text { landmark }\end{array}$ \\
\hline Perimount & & $0.1413 \pm 0.3116$ \\
\hline CE SAV (A) & 0.3227 & $0.11223 \pm 0.2021$ \\
\hline CE SAV (B) & 0.2306 & $0.1648 \pm 0.2330$ \\
\hline
\end{tabular}


Table 4. Registration errors with the ICP (RMS) and MHD methods for the four surgically explanted valves (Medtronic Mosaic, Carpentier Edwards Perimount, Carpentier Edwards Supra annular valve)

\section{Figures captions}

Figure 1. Fluoroscopic view during a valve-in-valve procedure. The transcatheter heart valve (blue arrow) is inserted inside the degenerated tissue valve (white arrow). The optimal level of implantation (dashed line) is determined according to the type of degenerated tissue valve and the used THV.

Figure 2. Segmentation of an undeteriorated Mosaic valve (Medtronic, Minneapolis, USA). The radiopaque landmarks are manually marked over the three radiopaque eyelets.

Figure 3. Alignment result using ICP algorithm on the Magna Ease 21mm (A) case. First row: Undeteriorated BVS in magenta. Degenerated BVS in blue. Second row: The undeteriorated BVS is colored with the approximate distance and the histogram of distances.

Figure 4. Alignment result using ICP algorithm on the Trifecta 23mm (A) case. First row: Undeteriorated BVS in magenta. Degenerated BVS in blue. Second row: The undeteriorated BVS is colored with the approximate distance and the histogram of distances.

Figure 5. Alignment result using ICP algorithm on the Epic 21mm (A) case. First row: Undeteriorated BVS in magenta. Degenerated BVS in blue. Second row: The undeteriorated BVS is colored with the approximate distance and the histogram of distances.

Figure 6. Alignment result using ICP algorithm on the Perimount 25 (A) case. First row: Undeteriorated BVS in magenta. Degenerated BVS in blue. Second row: The undeteriorated BVS is colored with the approximate distance and the histogram of distances. 


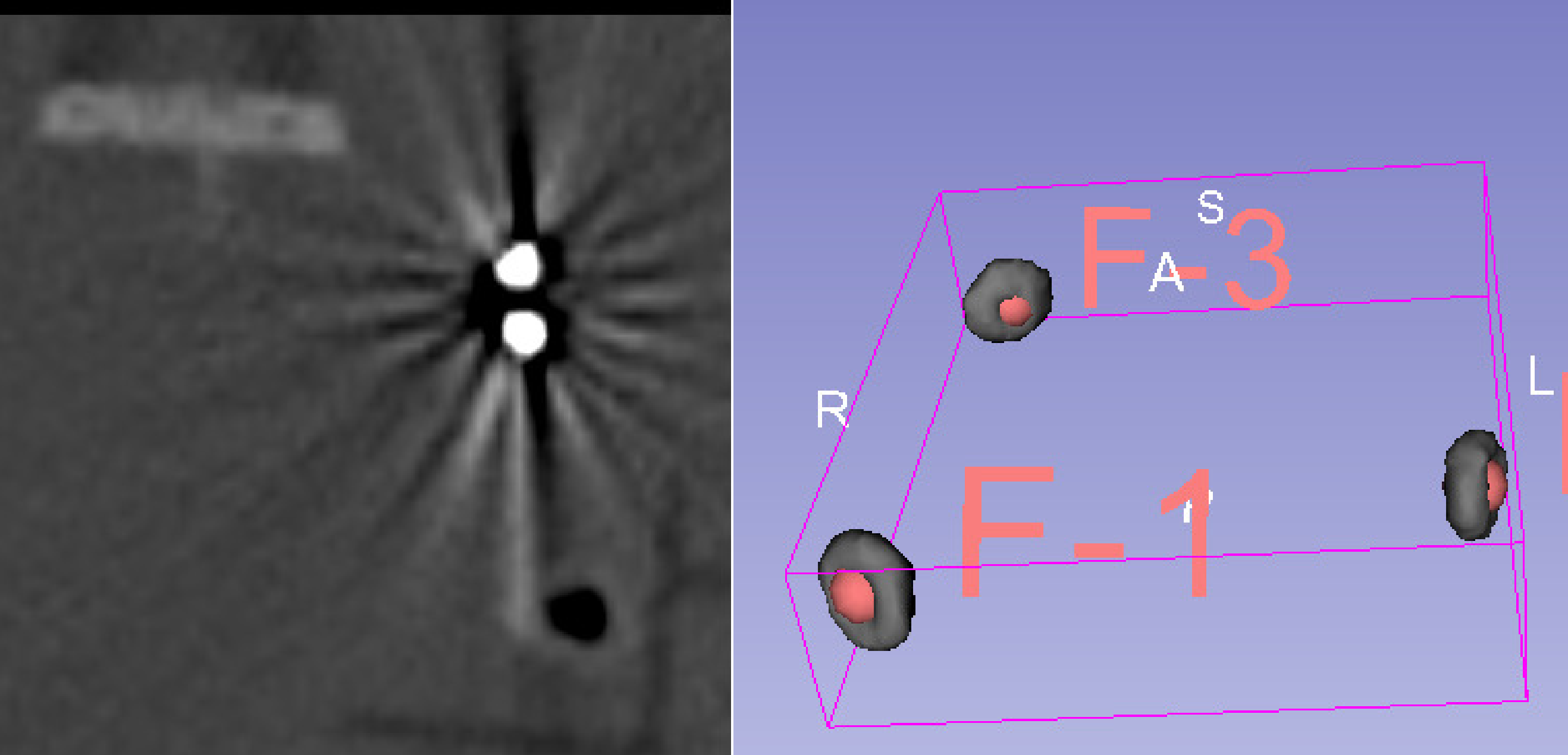

B: mosaic_25mm_1
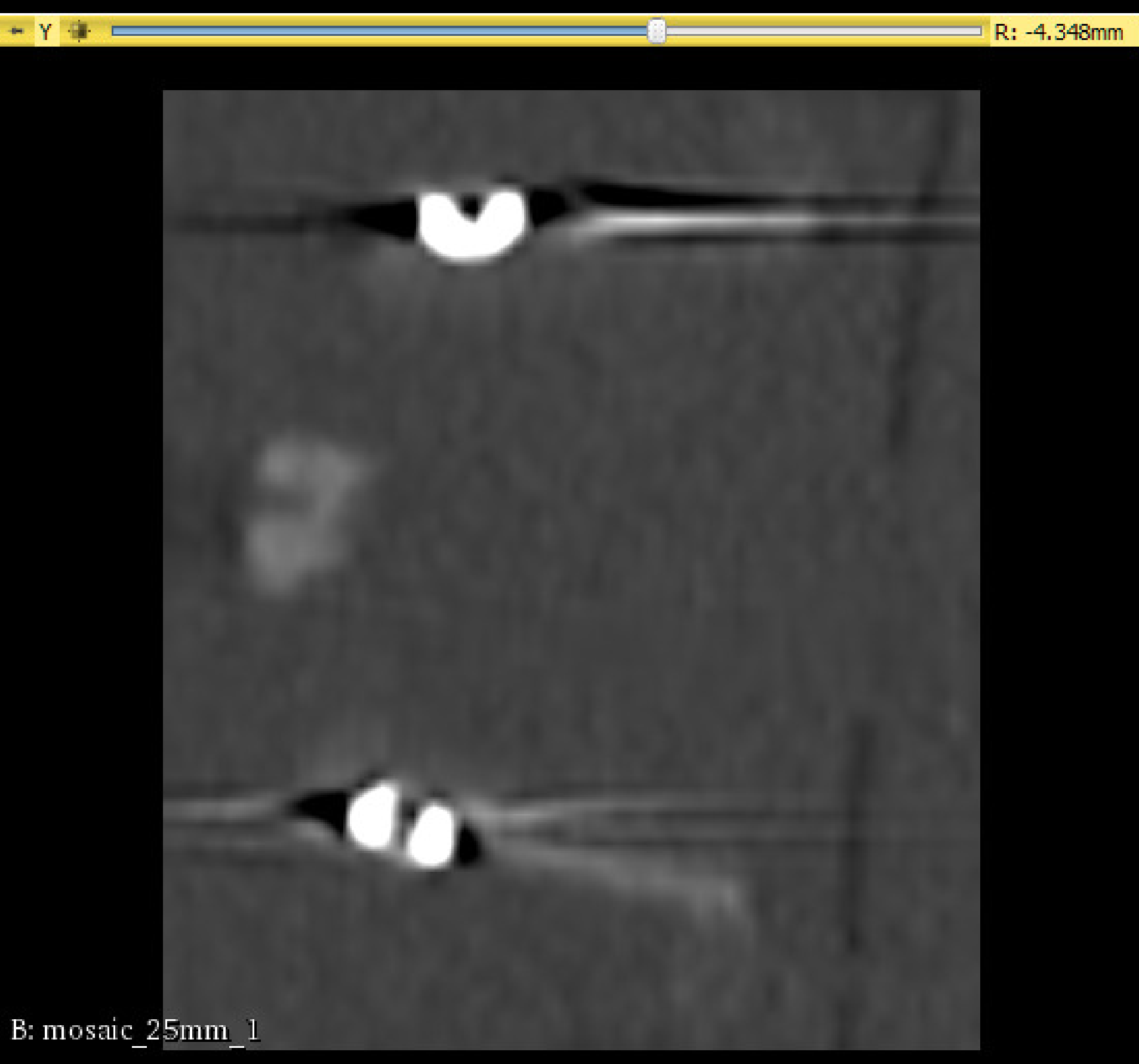

R. $-4.348 \mathrm{~mm}$ 


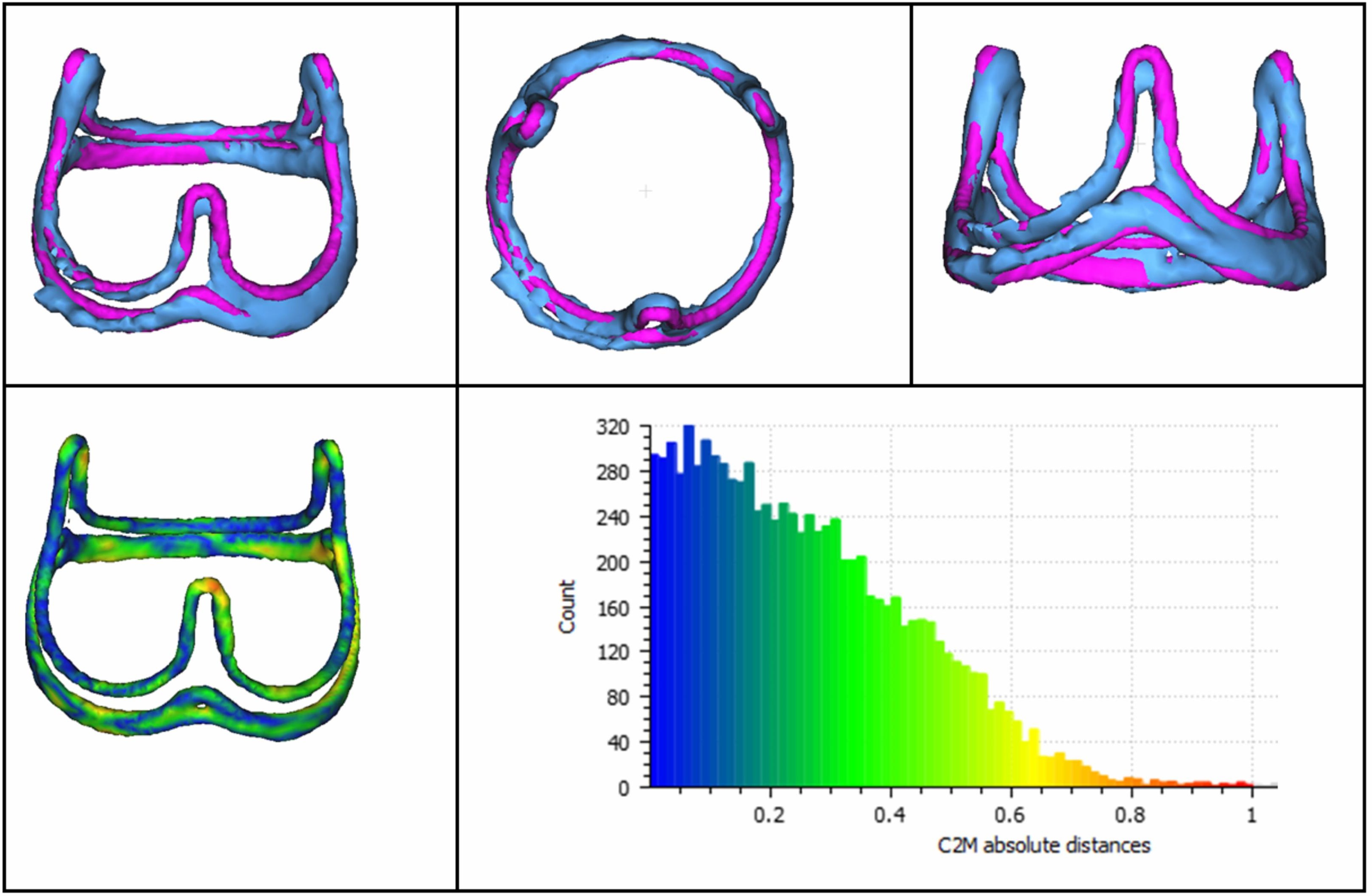




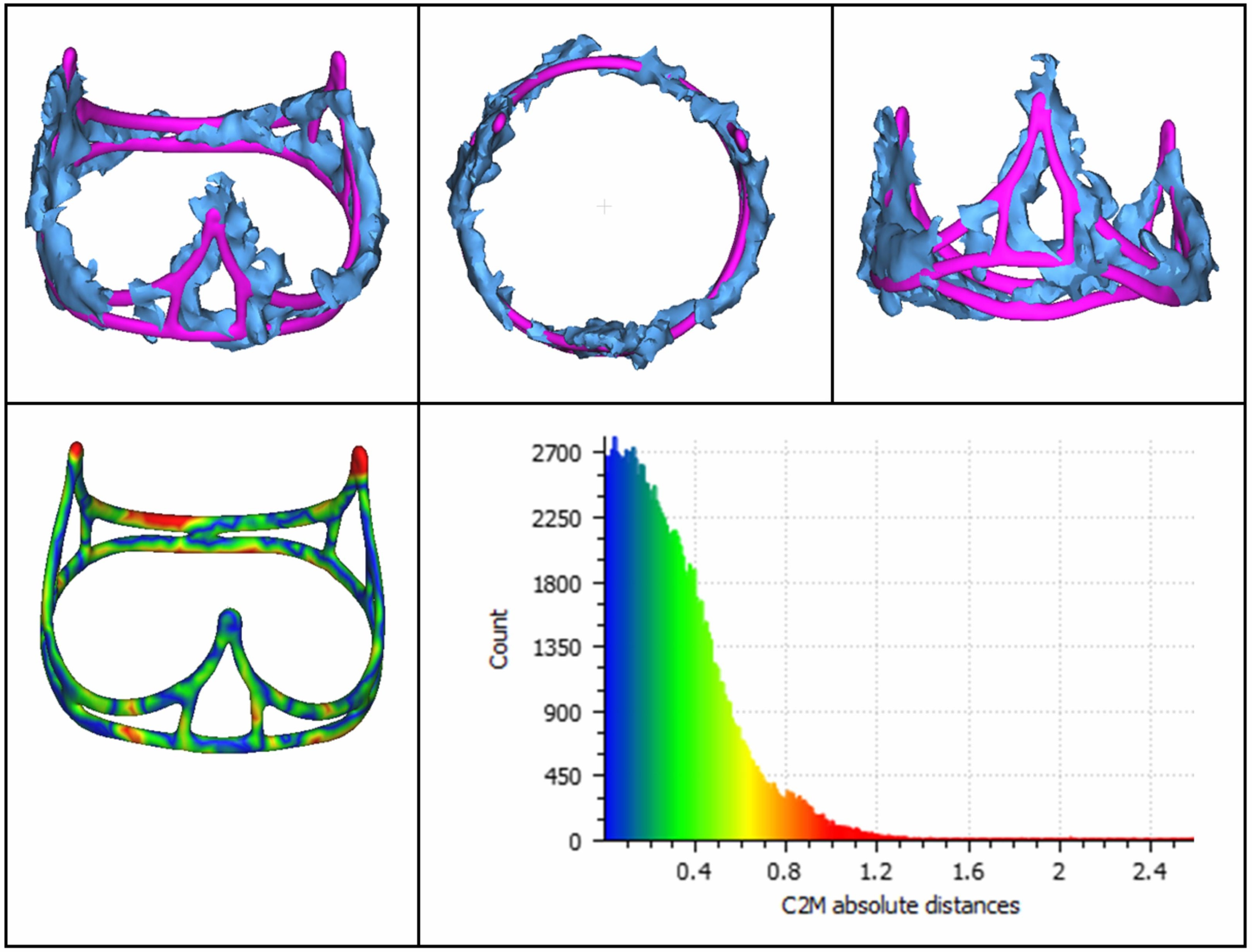


<smiles>C=Cc1ccccc1</smiles> 


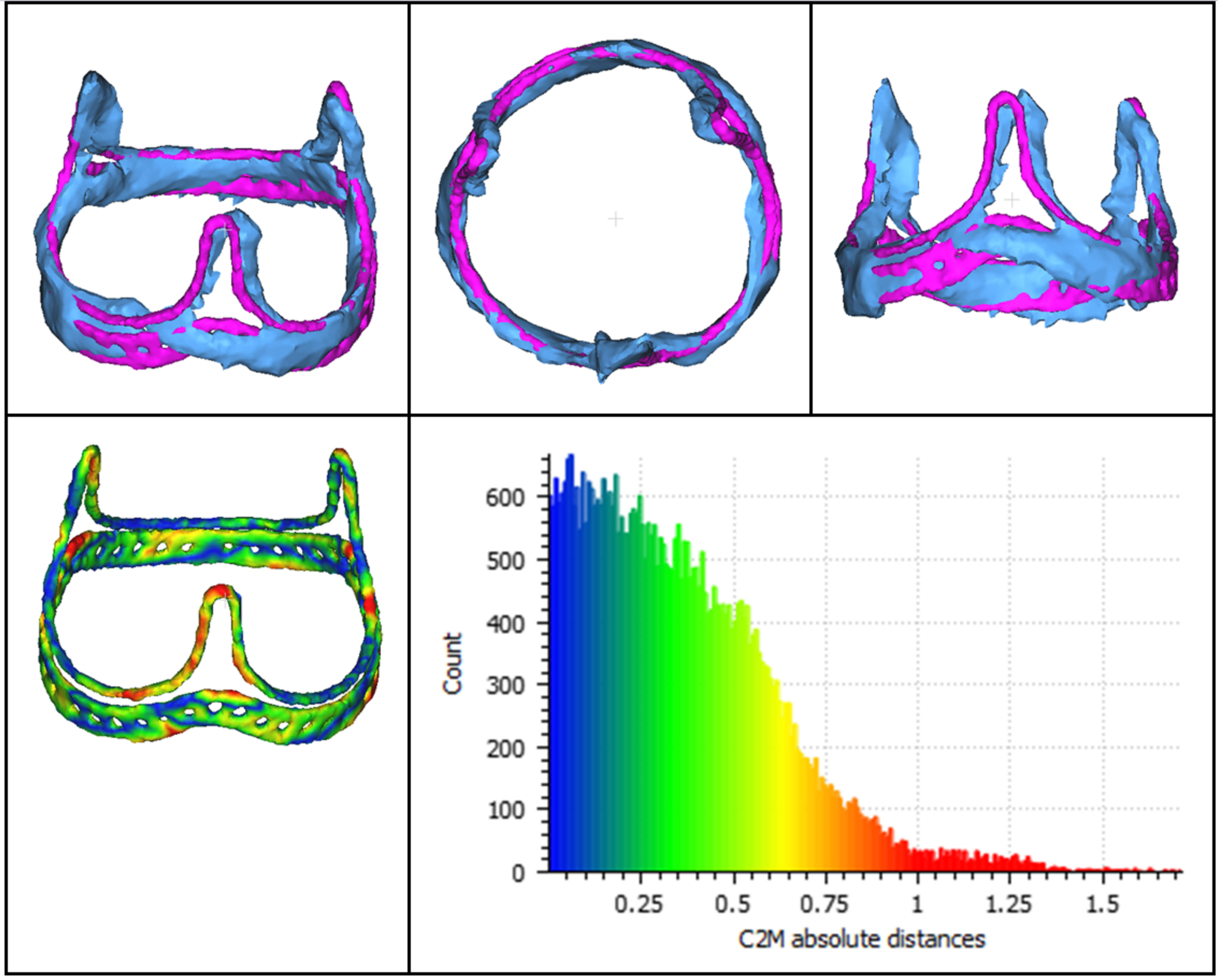

